\title{
Long noncoding RNA MAFG-AS1 promotes proliferation, migration and invasion of hepatocellular carcinoma cells through downregulation of miR-6852
}

\author{
HUI OUYANG ${ }^{1}$, LI ZHANG $^{2}$, ZHEN XIE $^{3}$ and SIMIN MA ${ }^{4}$ \\ ${ }^{1}$ Department of Cardiology, Tongji Hospital; ${ }^{2}$ Department of Neurology, The Central Hospital of Wuhan; \\ ${ }^{3}$ Department of Otorhinolaryngology, Union Hospital; ${ }^{4}$ Department of Nosocomial Infection Management, \\ Tongji Hospital, Tongji Medical College, Huazhong University of Science and Technology, Wuhan, Hubei 430030, P.R. China
}

Received November 13, 2018; Accepted June 27, 2019

DOI: $10.3892 /$ etm.2019.7850

\begin{abstract}
Long noncoding RNAs (1ncRNAs) have been shown to participate in the development and progression of a number of different types of cancer, including hepatocellular carcinoma (HCC). A recent report has indicated that lncRNA MAFG-antisense 1 (AS1) promotes colorectal cancer. However, the role of MAFG-AS1 in other types of cancer remains unclear. The aim of the present study was to examine the effect of IncRNA MAFG-AS1 in HCC. Based on The Cancer Genome Atlas database and reverse transcription-quantitative PCR results, it was determined that lncRNA MAFG-AS1 expression was increased in HCC tissues and cell lines. Following knockdown of lncRNA MAFG-AS1, a Cell Counting Kit-8 assay and Transwell assay demonstrated that the proliferation, migration and invasion of HCC cell lines were significantly inhibited. It was additionally demonstrated that there was a negative regulatory association between IncRNA MAFG-AS1 and miR-6852. Inhibition of miR-6852 increased proliferation, migration and invasion of HCC cell lines. LncRNA MAFG-AS1 promoted HCC development by dampening miR-6852 function and may thus be a novel target for treating patients with HCC.
\end{abstract}

\section{Introduction}

Hepatocellular carcinoma (HCC) refers to malignant tumors of the liver, including primary and metastatic HCC (1). In recent years, the incidence of HCC has increased on a global scale, ranking as the fifth most common malignant tumors (2).

Correspondence to: Dr Hui Ouyang, Department of Cardiology, Tongji Hospital, Tongji Medical College, Huazhong University of Science and Technology, 1095 Jiefang Road, Wuhan, Hubei 430030, P.R. China

E-mail: huiouyang1@163.com

Key words: long non-coding RNA, MAFG-antisense 1, hepatocellular carcinoma, microRNA-6852
The number of cases of HCC in China accounted for $\sim 55 \%$ of the world's total occurrence and presented with a high-risk of mortality (3). At present, surgical treatments, including liver resection and transplantation, are the primary means of treating patients with HCC (4). However, existing treatment techniques frequently fail to achieve favorable results due to a high incidence of metastasis and recurrence (5). Therefore, the underlying biological mechanisms need to be determined to develop improved therapeutic options.

At present, targeted therapy has been widely researched. In April 2018, antisense nucleic acid drug 'CT102 for injection' entered clinical trials in China and provided a new gene-targeted therapy for patients with HCC (6). Long noncoding RNAs (lncRNAs) are a group of noncoding RNAs over 200 nucleotides in length with little or no protein-coding ability (7). Various lncRNAs have been demonstrated to participate in the pathogenesis of $\operatorname{HCC}(7,8)$. In addition, lncRNA overexpression in HCC was closely related with other types of cancer, including colorectal cancer, gastric cancer, colorectal cancer and osteosarcoma (9). Xu et al (10) showed that lncSHRG promotes HCC progression through the transcription cofactor HES-6 pathway. Huang et al (11) found that lncAKHE contributes to HCC migration and invasion by activating the NOTCH signaling pathway. Upregulation of histone $\mathrm{H} 2 \mathrm{~B}$ ubiquitin ligase complex expression resulted in an interaction with miR-372, augmenting the cAMP-dependent protein kinase pathway and thus, promoting hepatocellular carcinoma (8). Furthermore, lncRNA metallothionein 1D, pseudogene was found to inhibit the expression of forkhead box (Fox)A1 in HCC cells by negatively regulating transcriptional coactivator YAP1 and runt-related transcription factor 2 (12). Various IncRNAs can regulate several biological processes in tumor cells, including apoptosis, proliferation, migration and invasion (12). Therefore, it is important to investigate and understand the functions of the numerous dysregulated lncRNAs in cancer.

LncRNA MAFG-antisense 1 (AS1) has been screened as a novel target for treating patients with cancer (13). Zhang et al (13) confirmed that IncRNA MAFG-AS1 influenced the proliferation of osteosarcoma cells and also regulated the expression level of various downstream proteins. 
Cui et al (14) showed that MAFG-AS1 promotes colorectal cancer development. Additionally, Jia et al (15) determined that MAFG-AS1 promotes metastasis of lung cancer (15). However, the molecular mechanism of IncRNA MAFG-AS1 in other types of cancer is still unknown. In the present study, the role of IncRNA MAFG-AS1 in HCC was explored, and potential regulatory factors and associated mechanisms were examined. LncRNA MAFG-AS1 may be a potentially novel target for treating patients with HCC.

\section{Materials and methods}

The Cancer Genome Atlas (TCGA) dataset analysis and cell culture. Two sets of HCC data were downloaded from the The Cancer Genome Atlas (TCGA) database (tcga-data.nci.nih. gov/tcga) with one set containing $40 \mathrm{HCC}$ samples and 25 controls, and the other containing 200 tumor samples and 50 controls. The human HCC cells (Hep3B and Huh7) and a human liver cell line (L02) were purchased from Shanghai Institute of Biochemistry and Cell Biology, and cultured using DMEM medium (Invitrogen; Thermo Fisher Scientific, Inc.) supplemented with 10\% FBS (Invitrogen; Thermo Fisher Scientific, Inc.) with $100 \mathrm{U} / \mathrm{ml}$ penicillin (Invitrogen; Thermo Fisher Scientific, Inc.) and streptomycin $(100 \mu \mathrm{g} / \mathrm{ml}$; Invitrogen; Thermo Fisher Scientific, Inc.) in an incubator with $5 \% \mathrm{CO}_{2}$ at $37^{\circ} \mathrm{C}$.

Reverse transcription-quantitative PCR (RT-qPCR). RT-qPCR was used to detect the expression level and distribution of MAFG-AS1. The cultured cells were digested and centrifuged at $4^{\circ} \mathrm{C}$ for $2 \mathrm{~min}$ at $3,000 \mathrm{x}$ g. Total RNA was extracted using TRlzol ${ }^{\circledR}$ reagent according to the manufacturer's protocol (Invitrogen; Thermo Fisher Scientific, Inc.) and quantified using NanoDrop 2000 (Thermo Fisher Scientific, Inc.). A total $1 \mathrm{mg}$ RNA was used for cDNA synthesis using PrimeScript RT Master mix system (Takara Biotechnology Co., Ltd.) according to the manufacturer's protocol. The reaction was performed with incubation at $42^{\circ} \mathrm{C}$ for $1 \mathrm{~h}$, and the enzyme was subsequently inactivated by incubation at $85^{\circ} \mathrm{C}$ for $5 \mathrm{~min}$. The total reaction volume was $20 \mu \mathrm{l}$ and the mixture was prepared as previously described (16). For qPCR, the PCR solution contained $1 \mathrm{ml}$ cDNA, $1 \mathrm{ml}$ primers and $10 \mathrm{ml} \mathrm{SYBR}$ Green and $5 \mathrm{ml} \mathrm{RT-qPCR} \mathrm{Master} \mathrm{mix} \mathrm{(all} \mathrm{from} \mathrm{Invitrogen;} \mathrm{Thermo}$ Fisher Scientific, Inc.). The final volume was adjusted to $20 \mu \mathrm{l}$ using RNase-free water. The amplification was carried out in an ABI FAST 7500 system (Applied Biosystems; Thermo Fisher Scientific Inc.). The relative expression level of each gene was normalized to U6 and calculated using the $2^{-\Delta \Delta \mathrm{Cq}}$ method (17). The thermocycling conditions were as follows: initial denaturation step of $94^{\circ} \mathrm{C}$ for $2 \mathrm{~min}$, followed by 30 cycles of $94^{\circ} \mathrm{C}$ for $30 \mathrm{sec}, 59^{\circ} \mathrm{C}$ for $30 \mathrm{sec}$ and $72^{\circ} \mathrm{C}$ for $2 \mathrm{~min}$ with a final extension step at $72^{\circ} \mathrm{C}$ for $10 \mathrm{~min}$. Primers for PCR amplification were: MAFG-AS1 forward, 5'-ATGACGACCCCCAATAAA GGA-3' and reverse, 5'-CACCGACATGGTTACCAGC-3; miR-6852 forward, 5'-AACGAGACGACGACAGAC-3' and reverse, 5'-CCCTGGGGTTCTGAGGACATG-3'; U6 forward, 5'-AACGAGACGACGACAGAC-3' and reverse, 5'-GCAAAT TCGTGAAGCGTTCCATA-3'.

Transient transfection. The siRNA (50 $\mathrm{nM})$ targeting MAFG-AS1 (5'-GGGCAAUUCCAACCAAGAAAC-3'), negative control siRNA (50 nM; 5'-AAUUCUCCGAAC GUGUCACGU-3'), miR-6852 mimics (5'-CCCUGGGGU UCUGAGGACAUG-3'; 50 nM), miR-6852 inhibitors (5'-CAUGUCCUCAGAACCCCAGGG-3'; $50 \mathrm{nM}$ ), inhibitor negative controls (5'-GCGUAACUAAUACAUCGGAUU CGU-3'; $50 \mathrm{nM}$ ) and mimic negative controls (5'-ACAUCU GCGUAAGAUUCGAGUCUA-3'; $50 \mathrm{nM}$ ) were obtained from Guangzhou RiboBio Co., Ltd. For MAFG-AS1 overexpression, the sequence of MAFG-AS1 was inserted into a pcDNA3.1 vector (Invitrogen; Thermo Fisher Scientific, Inc.). The cultured Hep3B and Huh7 cells were transfected using Lipofectamine $^{\circledR} 2000$ (Invitrogen; Thermo Fisher Scientific, Inc.) according to the manufacturer's instructions. Following $48 \mathrm{~h}$ of incubation, the transfection efficiency was analyzed by RT-qPCR.

Cell Counting Kit-8 (CCK8) assay. CCK8 assay was used to analyze the proliferation of HCC cell lines. The cells transfected for $48 \mathrm{~h}$ were plated at a density of $2 \times 10^{3}$ cells per well in a 24-well plate, and three replicate wells were used for each condition. The cells were cultured at $37^{\circ} \mathrm{C}$ in a $5 \% \mathrm{CO}_{2}$ incubator. Cell proliferation assays were performed at 0, 24, 48 and $72 \mathrm{~h}$. A total of $10 \mathrm{ml} \mathrm{CCK8}$ reagent was added to each well, incubated for 4-h at room temperature and the absorbance was measured at $450 \mathrm{~nm}$ (A) using a microplate reader.

Transwell migration and invasion assays. As described previously (18), $2 \times 10^{4}$ cells per well were cultured in the upper Transwell chamber of a 24-well plate (18). Matrigel (BD Biosciences) was used to coat the upper side of the membrane at $37^{\circ} \mathrm{C}$ for $30 \mathrm{~min}$. A total of $2 \times 10^{4}$ cells cultured in the upper chamber were placed in $200 \mu \mathrm{l} \mathrm{FBS-free} \mathrm{DMEM} \mathrm{(Invitrogen;}$ Thermo Fisher Scientific, Inc.) and $600 \mu \mathrm{l}$ DMEM with 10\% FBS was placed in the lower chamber. After a 17-h incubation, the cells were fixed in $70 \%$ methanol at room temperature for $30 \mathrm{~min}$ and subsequently stained with $0.1 \%$ crystal violet at room temperature for $30 \mathrm{~min}$. The cells on the lower chamber were counted using light microscopy to calculate the invasion at x200 magnification. Five random fields of views were counted.

The experimental procedures of the migration assay were the same as invasion assay except that Matrigel was not used.

Luciferase reporter assay. Bioinformatics analysis and luciferase reporter assay were used to screen binding sites and confirm the target of miR-6852, respectively. By using the miRDB tool (http://mirdb.org/miRDB/index.html), miR-6852 was identified as the most likely candidate target of MAFG-AS1. The 3' end of MAFG-AS1 containing the miR-6852 binding site was cloned into a luciferase reporter vector. A MAFG-AS1 3' UTR wild type (WT) plasmid (MAFG-AS1 3' UTR-WT) was constructed based on the $3^{\prime}$-end primer sequence of MAFG-AS1. Using the MAFG-AS1 3' UTR-WT plasmid, a binding site was mutated to construct a MAFG-AS1 3' UTR mutant (MUT) plasmid (MAFG-AS12 3' UTR-MUT). The construction and sequencing of the plasmid was performed by Sangon Bioengineering Co., Ltd. The constructed luciferase reporter plasmids pmirGLO-MAFG-AS1-WT, pmirGLO-MAFG-AS1-MUT and miR-6852 mimics, mimics negative control were co-transfected into Hep3B and Huh7 

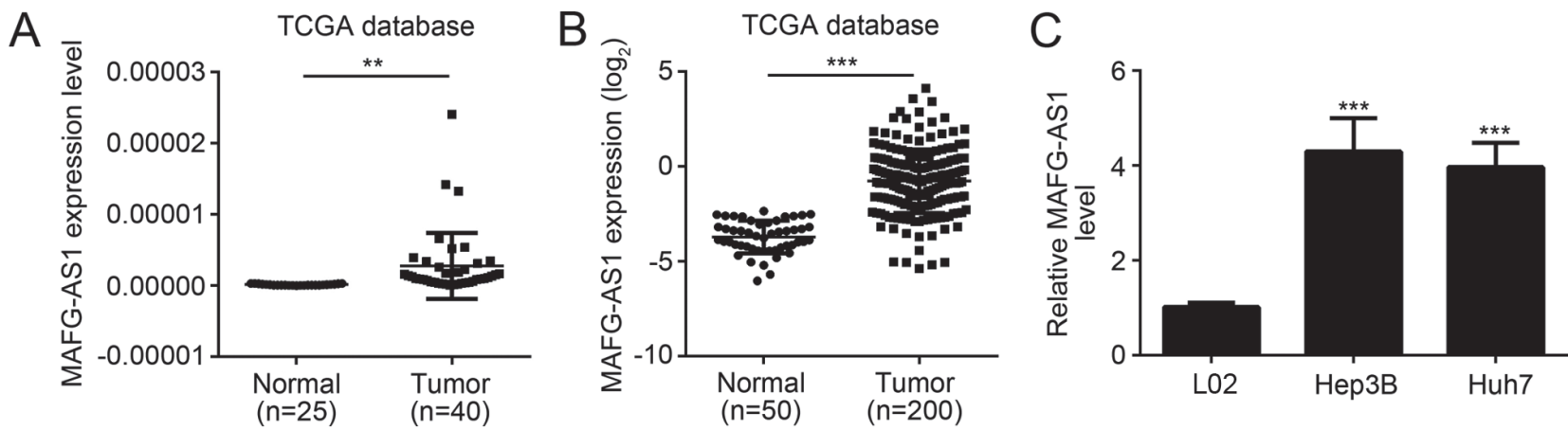

Figure 1. MAFG-AS1 expression is higher in HCC tissues and cell lines. (A) Based on the TCGA database, MAFG-AS1 expression in HCC tissues was significantly higher compared with normal tissues. ${ }^{* *} \mathrm{P}<0.01$ with comparisons indicated by lines. (B) Based on the other set of data in the TCGA database, MAFG-AS1 expression in HCC tissues was significantly higher compared with normal tissues. ${ }^{* * *} \mathrm{P}<0.001$ comparisons indicated by lines. (C) Reverse transcription-quantitative PCR results revealed that MAFG-AS1 expression was significantly higher in HCC cell lines compared with the control cell line. ${ }^{* * *} \mathrm{P}<0.001$ vs. L02. HCC, hepatocellular carcinoma; TCGA, The Cancer Genome Atlas; AS, antisense.
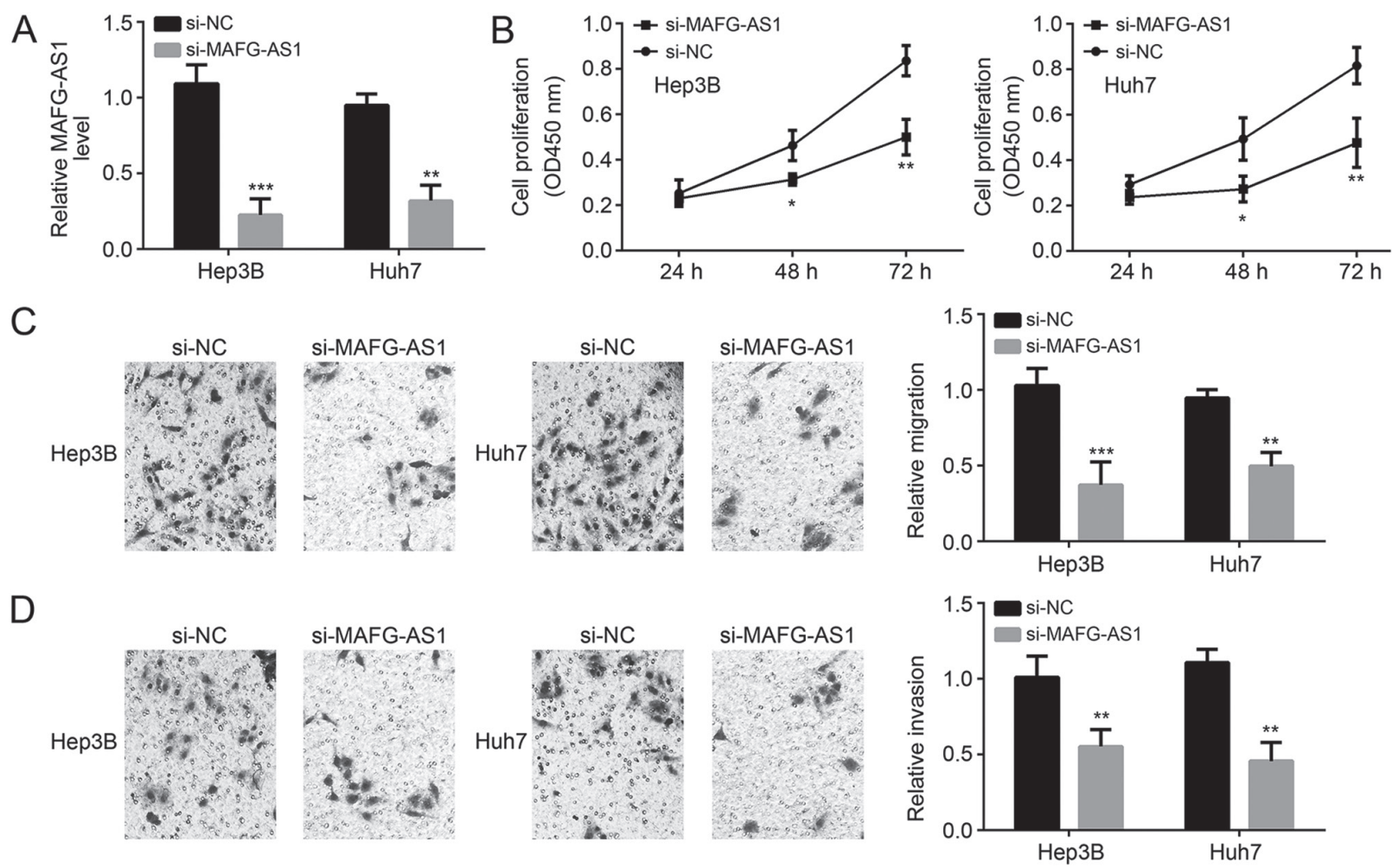

Figure 2. MAFG-AS1 promotes proliferation, migration and invasion of HCC cell lines. (A) MAFG-AS1 was successfully knocked down in HCC cell lines using siRNA. ${ }^{* *} \mathrm{P}<0.01,{ }^{* * *} \mathrm{P}<0.001$. (B) Cell Counting Kit-8 experiment results showed that MAFG-AS1 knockdown decreased proliferation of HCC cells. ${ }^{*} \mathrm{P}<0.05,{ }^{* *} \mathrm{P}<0.01$. Transwell assays confirmed that MAFG-AS1 knockdown inhibited (C) migration and (D) invasion of HCC cell lines (magnification, $\left.\mathrm{x} 100\right)$. ${ }^{* *} \mathrm{P}<0.01$ and ${ }^{* * *} \mathrm{P}<0.001$ vs. si-NC. HCC, hepatocellular carcinoma; AS, antisense; si, small interfering; NC, negative control; OD, optical density.

cell lines using Lipofectamine ${ }^{\circledR} 2000$ (Invitrogen; Thermo Fisher Scientific, Inc.). After $48 \mathrm{~h}$, the luciferase activity was measured using a dual luciferase activity assay kit (Promega Corporation) and results were normalized to Renilla luciferase activity.

Western blotting. HCC cells were lysed using radioimmunoprecipitation assay buffer (Thermo Fisher Scientific, Inc.) and the total protein lysates were obtained. Protein concentration was determined using a bicinchoninic acid assay. Total protein $(20 \mu \mathrm{g})$ was separated using SDS-PAGE on a 4-20\% gel and then transferred to polyvinylidene difluoride membranes. Membranes were subsequently blocked in 5\% non-fat milk/Tris-buffered saline containing $0.1 \%$ Tween-20 at $25^{\circ} \mathrm{C}$ for $1 \mathrm{~h}$ and then incubated at $4^{\circ} \mathrm{C}$ overnight with the primary antibodies. The following primary antibodies were used: Anti-PI3K (1:2,000; cat. no. 4292), anti-p-PI3K (1:2,000; cat. no. 4228), anti-p-STAT3 (1:2,000; cat. no. 9145), anti-STAT3 (1:2,000; cat. no. 12640), anti-MYC (1:2,000; cat. no. 5605) and GAPDH (1:2,000; cat. no. 5174) all from Cell Signaling Technology, Inc. Subsequently, the membranes were incubated with goat anti-rabbit horseradish peroxidase-conjugated secondary antibody (1:2,000; cat. no. 7074; Cell Signaling Technology, Inc.) for $2 \mathrm{~h}$ at $25^{\circ} \mathrm{C}$. Signals were 

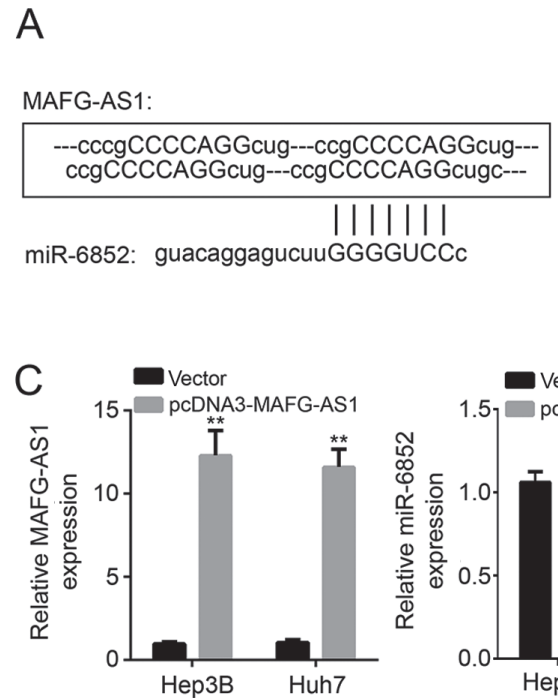
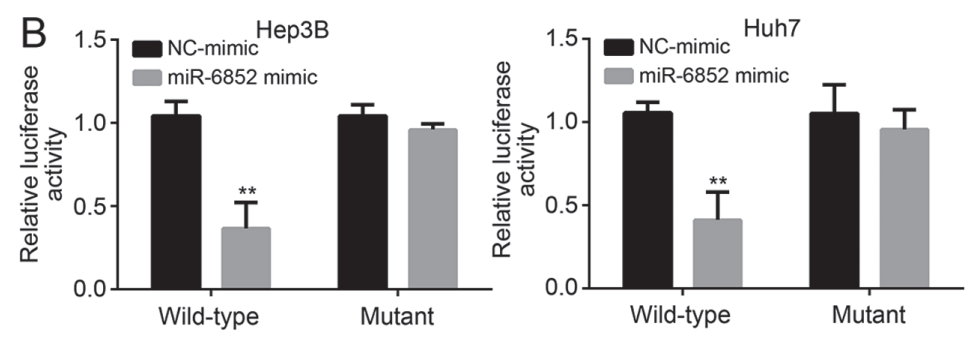

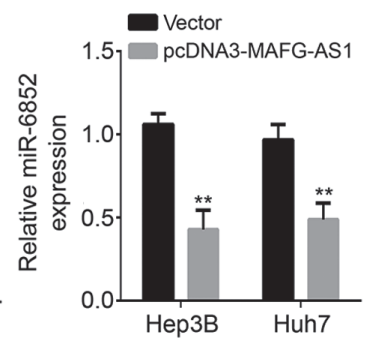

Figure 3. MAFG-AS1 targets and negatively regulates miR-6852. (A) Bioinformatics analysis showed that MAFG-AS1 has four potential binding sites for miR-6852. (B) Luciferase reporter assay revealed that overexpression of miR-6852 inhibited the activity of the wild-type MAFG-AS1 reporter in the hepatocellular carcinoma cell lines. ${ }^{* *} \mathrm{P}<0.01$ vs. NC-mimic. (C) Reverse transcription-quantitative PCR analysis revealed that overexpression of MAFG-AS1 decreased the expression levels of miR-6852. ${ }^{* *} \mathrm{P}<0.01$ vs. vector. AS, antisense; miR, microRNA; NC, negative control.

visualized using the Pierce ${ }^{\mathrm{TM}}$ ECL Western Blotting Substrate (Thermo Fisher Scientific, Inc.). Densitometry analysis was performed using ImageJ version 1.41 (National Institutes of Health).

Statistical analysis. All data were analyzed using SPSS 18.0 statistical software (SPSS, Inc.). All data were expressed as the mean \pm standard deviation of at least three repeats. A Student's t-test was performed for comparison between two groups. A one-way ANOVA followed by Tukey's post-hoc test was performed for comparisons between multiple groups. $\mathrm{P}<0.05$ was considered to indicate a statistically significant difference.

\section{Results}

Expression levels of MAFG-ASI in HCC tissues and cell lines. Based on two sets of data obtained from TCGA database, the expression of MAFG-AS1 in HCC tissues was significantly increased compared with the normal controls $(\mathrm{P}<0.01$; Fig. 1A and B). Similarly, the expression of MAFG-AS1 in Hep3B and Huh7 cell lines were significantly higher compared with the L02 cell line $(\mathrm{P}<0.01$; Fig. 1C). MAFG-AS1 expression was increased in both HCC tissues and cell lines compared with their respective controls.

MAFG-AS1 knockdown inhibits proliferation, invasion and migration of HCC cells. The relative MAFG-AS1 mRNA expression levels were significantly decreased following MAFG-AS1 knockdown in both cell lines confirming transfection was successful (Fig. 2A). Following MAFG-AS1 knockdown, the proliferation, invasion and migration of both Hep3B and Huh7 cell lines were significantly inhibited (Fig. 2B-D) suggesting that MAFG-AS1 was a critical gene in pathogenesis of HCC.

Cellular localization and target of MAFG-AS1. A previous study demonstrated that MAFG-AS1 was primarily localized in the cytoplasm and inhibits miRNAs in colorectal cancer (14). Therefore, it was hypothesized that MAFG-AS1 may utilize a similar mechanism in HCC. Bioinformatics analysis was used to identify potential binding targets. The analysis indicated that miR-6852 ranked top among all the potential targets. MAFG-AS1 has four potential binding sites with miR-6852 (Fig. 3A). A luciferase reporter assay revealed that upregulation of miR-6852 decreased the luciferase activity of the wild-type MAFG-AS1 reporter in both HCC cell lines ( $\mathrm{P}<0.01$; Fig. 3B). Upregulation of MAFG-AS1 decreased the expression levels of miR-6852 ( $\mathrm{P}<0.01$; Fig. 3C). The above results demonstrate that MAFG-AS1 targets and negatively regulates miR-6852.

Inhibiting miR-6852 decreases proliferation, migration and invasion of HCC cells. miR-6852 was knocked-down using miR-6852 inhibitors in Hep3B and Huh7 cells (Fig. 4A). In the si-MAFG-AS1 and miR-6852 mimics groups, proliferation, migration and invasion of HCC cell lines were significantly decreased ( $\mathrm{P}<0.01$; Fig. 4B). However, inhibiting miR-6852 restored proliferation, migration and invasion of the HCC cell lines ( $\mathrm{P}<0.01$; Fig. $4 \mathrm{C}$ and $\mathrm{D})$. Taken together, these results demonstrated that MAFG-AS1 may promote HCC progression through inhibition of miR-6852. The effect of MAFG-AS1 on expression of members of the STAT3, Wnt $/ \beta$-catenin and PI3K/AKT signaling pathways were determined, which are related to proliferation, migration and invasion of HCC (19). Western blotting showed that MAFG-AS1 silencing only suppressed the activation of the PI3K/AKT signaling pathway (Fig. 5).

\section{Discussion}

Numerous 1 ncRNAs are aberrantly expressed in HCC, regulating various miRNAs and genes, and modulating a variety of biological processes (20-22). Therefore, it is possible that one or more of these IncRNAs may serve as a potential therapeutic target for treating patients with HCC. In the present study, 

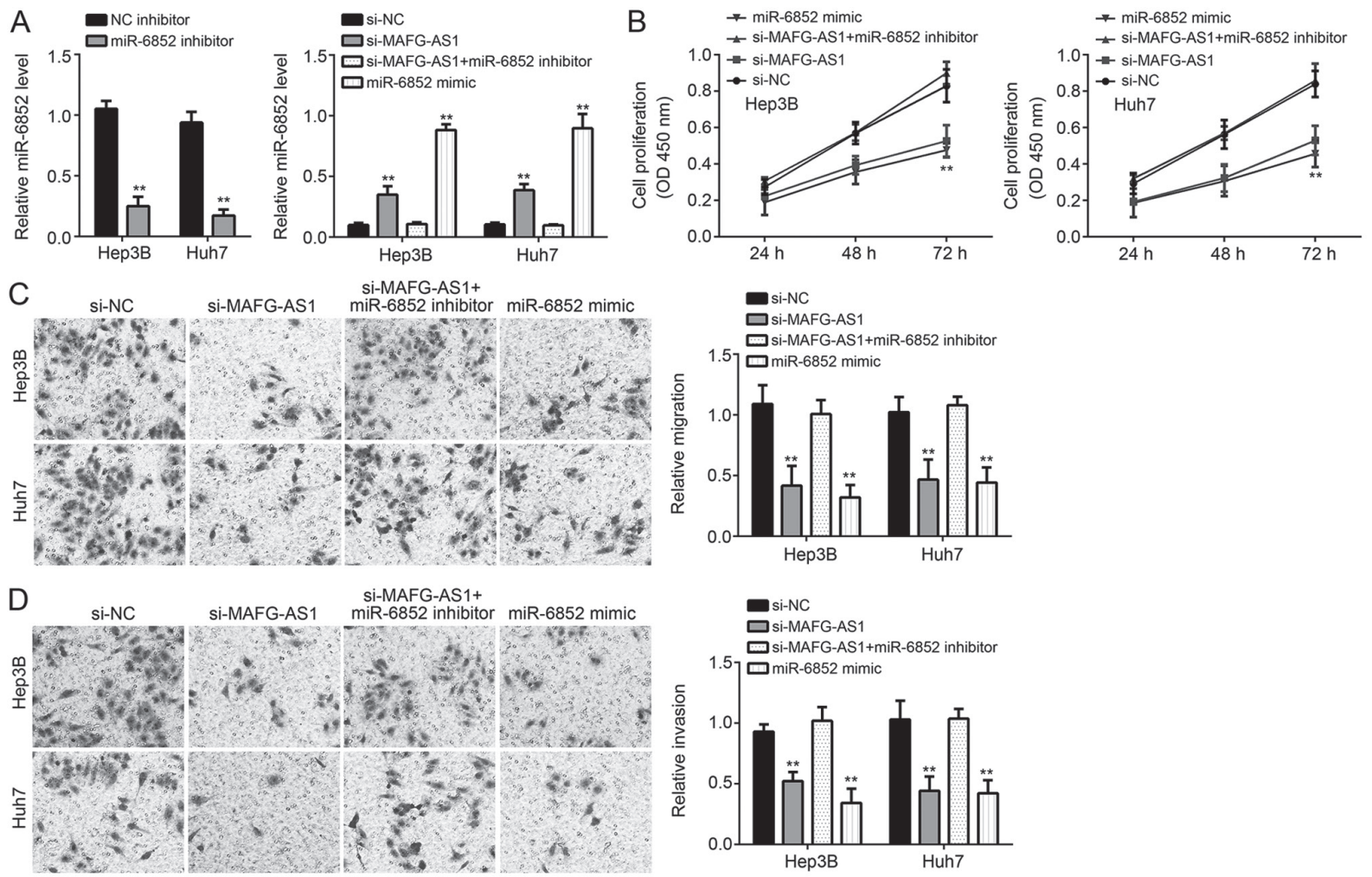

Figure 4. MAFG-AS1 knockdown inhibits cell proliferation, migration and invasion. (A) Reverse transcription-quantitative PCR analysis was used to determine the expression of miR-6852 in cells transfected with a miR-6852 inhibitor. ${ }^{* *} \mathrm{P}<0.01$ vs. NC inhibitor. (B) Cell proliferation was analyzed using a Cell Counting Kit- 8 assay. ${ }^{* *} \mathrm{P}<0.01$ vs. si-NC. Transwell assays were used to determine (C) migration and (D) invasion (magnification, $\left.\mathrm{x} 100\right)$ in the hepatocellular carcinoma cell lines transfected with a miR-6852 inhibitor, siRNA against MAFG-AS1 or both. ${ }^{* *} \mathrm{P}<0.01$ vs. si-NC. AS, antisense; miR, microRNA; si, small interfering; NC, negative control; OD, optical density.

A

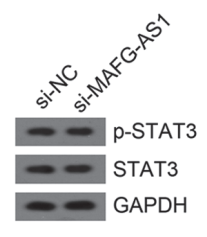

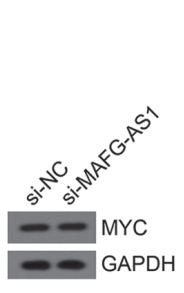

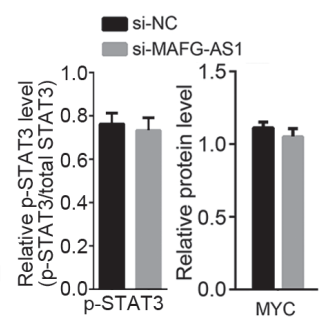

B

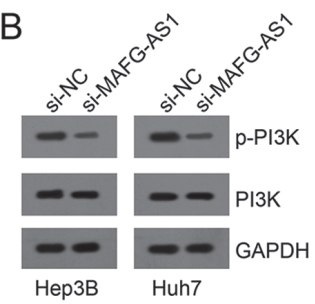

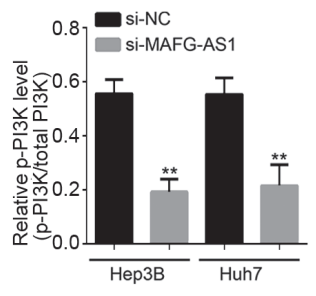

Figure 5. MAFG-AS1 silencing suppresses the activation of the PI3K/AKT signaling pathway. (A) Western blotting results and quantitative analysis of expression of p-STAT3 and MYC (a target of Wnt/ $\beta$-catenin pathway) in Hep3B cells. (B) Western blotting analysis and quantitative analysis of the expression levels of p-PI3K in Hep3B and Huh7 cells. ${ }^{* *} \mathrm{P}<0.01$ vs. si-NC. AS, antisense; p-, phosphorylated; si, small interfering; NC, negative control.

the functional effects and potential underlying mechanism of lncRNA MAFG-AS1 in HCC was examined. Based on the TCGA database and RT-qPCR results, lncRNA MAFG-AS1 was highly expressed in HCC tissues and cell lines. After knockdown of lncRNA MAFG-AS1, the proliferation, migration and invasion of HCC cell lines were significantly decreased. Interestingly, IncRNA MAFG-AS1 and miR-6852 were demonstrated to exhibit a reciprocally negative regulatory association with each other. By inhibiting the expression of miR-6852, MAFG-AS1 promoted proliferation, migration and invasion of HCC cells.

Zhang et al (23) processed a regulatory network analysis of lncRNAs in colorectal cancer and showed that lncRNA MAFG-AS1 was upregulated in this disease (23). In addition, high-throughput data analysis and in vitro experiments confirmed that lncRNA MAFG-AS1 was highly expressed and affects the proliferation of osteosarcoma cells (13). Similarly, lncRNA MAFG-AS1 expression was also upregulated in HCC tissues and primarily distributed in the cytoplasm of HCC cells in the present study. After knockdown of lncRNA MAFG-AS1, the proliferation, migration and invasion of HCC cell lines were significantly inhibited. Therefore, lncRNA MAFG-AS1 may serve a critical role in pathogenesis of HCC.

LncRNA MAFG-AS1 was found to negatively regulate the expression of miR-6852 through four potential binding sites, and further influence the proliferation, migration and invasion of HCC cell lines. A luciferase reporter assay demonstrated that overexpression of miR-6852 inhibited the activity of the wild-type MAFG-AS1 reporter. As shown in previous studies, abnormal expression of miR-6852 could regulate the expression of FoxM1, and thus influence processes of cell cycle arrest and necrosis in cervical cancer cells (24). Kopanja et al (25) 
found that upregulation of FoxM1 was associated with a poor prognosis in patients with HCC possibly by participating in the Ras signaling pathway. In colorectal cancer, miR-6852 was confirmed to target lncRNA transcription factor 7 (TCF7), and suppress tumor metastasis and growth (26). Furthermore, miR-6852 modulated cell invasion and proliferation by regulating the expression of FoxJ1 (27). LncRNA TCF7 could activate the Wnt signaling pathway and promote self-renewal of HCC cells (28). In the present study, miR-6852 was inhibited by MAFG-AS1, and was a critical factor for the proliferation, migration and invasion of HCC cell lines. Notably, MAFG-AS1 was also reported to prevent binding of miR-339-5p from MMP15 in non-small cell lung cancer (15). Whether MAFG-AS1 similarly suppressed miR-339-5p to upregulate MMP15 in HCC cells remains to be determined.

In the present study, the TCGA database was used to show the expression of IncRNA MAFG-AS1 in HCC tissues. TCGA was jointly developed by he National Cancer Institute and the National Human Genome Research Institute in 2006, and a total 36 types of cancer were examined (29). Large-scale sequencing-based genomic analysis technology was used to aid in understanding the molecular mechanisms of cancer (30). TCGA combined with other bioinformatics platforms may improve our understanding of the molecular basis of cancer and improve diagnosis, treatment and prevention (31). In the present study, results from the TCGA database analysis confirmed that IncRNA MAFG-AS1 was highly expressed in HCC tissues, it was also consistent with the results of the in vitro experiments.

In conclusion, lncRNA MAFG-AS1 was overexpressed in patients with HCC. Furthermore, overexpression of lncRNA MAFG-AS1 downregulated the expression of miR-6852 and thus increased the proliferation, migration and invasion of HCC cells. These results may improve our understanding of the molecular mechanisms underlying development and prognosis of HCC, and IncRNA MAFG-AS1 may be a valuable therapeutic target for treating patients with HCC.

\section{Acknowledgements}

Not applicable.

\section{Funding}

No funding was received.

\section{Availability of data and materials}

The datasets used and/or analyzed during the present study are available from the author on reasonable request.

\section{Authors' contributions}

HOY contributed to the conception and design of the present study. In addition, HOY analyzed and interpreted the results and wrote the manuscript. LZ, ZX and SM performed the experiments. All authors read and approved the final manuscript.

\section{Ethics approval and consent to participate}

Not applicable.

\section{Patient consent for publication}

Not applicable.

\section{Competing interests}

The authors declare that they have no competing interests.

\section{References}

1. Akyuz M, Yazici P, Yigitbas H, Dural C, Okoh A, Aliyev S, Aucejo F, Quintini C, Fung J and Berber E: Oncologic results of laparoscopic liver resection for malignant liver tumors. J Surg Oncol 113: 127-129, 2016.

2. Bai L, Liu Z, Fang Q, Yan Q, Shi O, Bao P, Mu L, Chen X and Zhang T: The trends and projections in the incidence and mortality of liver cancer in urban Shanghai: A population-based study from 1973 to 2020. Clin Epidemiol 10: 277-288, 2018.

3. Zhang SW, Zheng RS, Li N, Zeng HM, Dai Z, Zou XN and Chen WQ: Analysis and prediction of liver cancer incidence in China.Zhonghua Yu Fang Yi Xue Za Zhi 46: 587, 2012 (In Chinese).

4. Fuchs J, Cavdar S, Blumenstock G, Ebinger M, Schäfer JF, Sipos B and Warmann SW: POST-TEXT III and IV hepatoblastoma: Extended hepatic resection avoids liver transplantation in selected cases. Ann Surg 266: 318-323, 2017.

5. Min BS, Kim NK, Jeong HC and Chung HC: High levels of serum VEGF and TIMP-1 are correlated with colon cancer liver metastasis and intrahepatic recurrence after liver resection. Oncol Lett 4: 123-130, 2012.

6. Sadelain M, Brentjens R, Davila M, Riviere I, Wang X, Bartido S, Park J, Bouhassira D, Curran K, Chung S, et al: Abstract CT102: Efficacy and toxicity management of 19-28z CAR T cell therapy in B cell acute lymphoblastic leukemia. Cancer Res 74: CT102, 2014.

7. Li H, He Z, Gu Y, Fang L and Lv X: Prioritization of non-coding disease-causing variants and long non-coding RNAs in liver cancer. Oncol Lett 12: 3987, 2016.

8. Wang J, Liu X, Wu H, Ni P, Gu Z, Qiao Y, Chen N, Sun F and Fan Q: CREB up-regulates long non-coding RNA, HULC expression through interaction with microRNA-372 in liver cancer. Nucleic Acids Res 38: 5366-5383, 2010

9. Ma Z, Huang H, Xu Y, He X, Wang J, Hui B, Ji H, Zhou J and Wang K: Current advances of long non-coding RNA highly upregulated in liver cancer in human tumors. Onco Targets Ther 10: 4711-4717, 2017.

10. Xu YC, Liang CJ, Zhang DX, Li GQ, Gao X, Fu JZ, Xia F, Ji JJ, Zhang LJ, Li GM and Wu JX: LncSHRG promotes hepatocellular carcinoma progression by activating HES6. Oncotarget 8: 70630-70641, 2017.

11. Huang G, Jiang H, Lin Y, Wu Y, Cai W, Shi B, Luo Y, Jian Z and Zhou X: lncAKHE enhances cell growth and migration in hepatocellular carcinoma via activation of NOTCH2 signaling. Cell Death Dis 9: 487, 2018.

12. Wang J, Wang H, Zhang Y, Zhen N, Zhang L, Qiao Y, Weng W, Liu X, Ma L, Xiao W, et al: Mutual inhibition between YAP and SRSF1 maintains long non-coding RNA, Malat1-induced tumourigenesis in liver cancer. Cell Signal 26: 1048-1059, 2014.

13. Zhang $\mathrm{H}$, Ma Q, Yang T, Zhang T and Zhou Y: The expression and function of IncRNA MAFG-AS1 in osteosarcoma. J Modern Oncol: 3536-3540, 2018.

14. Cui S, Yang X, Zhang L, Zhao Y and Yan W: LncRNA MAFG-AS1 promotes the progression of colorectal cancer by sponging miR-147b and activation of NDUFA4. Biochem Biophys Res Commun 506: 251-258, 2018.

15. Jia YC, Wang JY, Liu YY, Li B, Guo H and Zang AM: LncRNA MAFG-AS1 facilitates the migration and invasion of NSCLC cell via sponging miR-339-5p from MMP15. Cell Biol Int 43: 384-393, 2019.

16. Ye B, Hu B, Zheng Z, Zheng R and Shi Y: The long non-coding RNA AK023948 enhances tumor progression in hepatocellular carcinoma. Exp Ther Med 14: 3658-3664, 2017.

17. Livak KJ and Schmittgen TD: Analysis of relative gene expression data using real-time quantitative PCR and the 2(-Delta Delta C(T)) method. Methods 25: 402-408, 2001.

18. Shin I, Kim S, Song H, Kim HR and Moon A: H-Ras-specific activation of Rac-MKK3/6-p38 pathway: Its critical role in invasion and migration of breast epithelial cells. J Biol Chem 280: 14675-14683, 2005. 
19. Zhu P, Liu Z, Zhou J and Chen Y: Tanshinol inhibits the growth migration and invasion of hepatocellular carcinoma cells via regulating the PI3K-AKT signaling pathway. Onco Targets Ther 12: 87-99, 2019.

20. Chen Z, Yu C, Zhan L, Pan Y, Chen L and Sun C: LncRNA CRNDE promotes hepatic carcinoma cell proliferation, migration and invasion by suppressing miR-384. Am J Cancer Res 6: 2299-2309, 2016.

21. She K, Huang J, Zhou H, Huang $T$, Chen $G$ and $\mathrm{He} J$ : IncRNA-SNHG 7 promotes the proliferation, migration and invasion and inhibits apoptosis of lung cancer cells by enhancing the FAIM2 expression. Oncol Rep 36: 2673, 2016.

22. Han F, Wang C, Wang Y and Zhang L: Long noncoding RNA ATB promotes osteosarcoma cell proliferation, migration and invasion by suppressing miR-200s. Am J Cancer Res 7: 770-783, 2017.

23. Zhang Y, Tao Y, Li Y, Zhao J, Zhang L, Zhang X, Dong C, Xie Y, Dai X, Zhang $X$ and Liao Q: The regulatory network analysis of long noncoding RNAs in human colorectal cancer. Funct Integr Genomics 18: 261-275, 2018.

24. Poudyal D, Herman A, Adelsberger JW, Yang J, Hu X, Chen Q, Bosche M, Sherman BT and Imamichi T: A novel microRNA, hsa-miR-6852 differentially regulated by Interleukin-27 induces necrosis in cervical cancer cells by downregulating the FoxM1 expression. Sci Rep 8: 900, 2018.

25. Kopanja D, Pandey A, Kiefer M, Wang Z, Chandan N, Carr JR, Franks R, Yu DY, Guzman G, Maker A and Raychaudhuri P: Essential roles of FoxM1 in Ras-induced liver cancer progression and in cancer cells with stem cell features. J Hepatol 63: 429-436, 2015.
26. Cui BH and Hong X: miR-6852 serves as a prognostic biomarker in colorectal cancer and inhibits tumor growth and metastasis by targeting TCF7. Exp Ther Med 16: 879-885, 2018.

27. YuH,Zhang J,WenQ,Dai Y,Zhang W,LiFandLiJ: MicroRNA-6852 suppresses cell proliferation and invasion via targeting forkhead box $\mathrm{J} 1$ in gastric cancer. Exp Ther Med 16: 3249-3255, 2018.

28. Wang Y, He L, Du Y, Zhu P, Huang G, Luo J, Yan X, Ye B, Li C, Xia P, et al: The long noncoding RNA lncTCF7 promotes self-renewal of human liver cancer stem cells through activation of Wnt signaling. Cell Stem Cell 16: 413-425, 2015.

29. Collins A: The Cancer Genome Atlas (TCGA) pilot project. Cancer Res 67, 2007.

30. Huang Z, Duan H and Li H: TCGA4U: A web-based genomic analysis platform to explore and mine TCGA genomic data for translational research. Stud Health Technol Inform 216: 658-662, 2015.

31. Levine D and Mardis E; T. Cancer Genome Atlas Research Network: Late-breaking abstract 4: The Cancer Genome Atlas (TCGA) project on endometrial carcinoma: Initial data and preliminary genomic analysis. Gynecol Oncol 125: 772, 2012.

c) (i) $(-)$ This work is licensed under a Creative Commons

CY NC ND Attribution-NonCommercial-NoDerivatives 4.0 International (CC BY-NC-ND 4.0) License. 